\title{
Boundaries of Varieties in Projective Manifolds
}

\author{
by \\ F. Reese Harvey and H. Blaine Lawson, Jr. ${ }^{1}$
}

\begin{abstract}
We give a characterization of the boundaries of holomorphic chains in complex projective space. This extends previous work of the authors and complements results of Dolbeault and Henkin.
\end{abstract}

\section{Table of Contents}

1. Introduction

2. Statement of the Problem

3. Discussion of the Main Result

4. Newton Hierarchies

5. Newton Hierarchies with Coefficients in a Vector Bundle

6. Vector Bundle-valued Currents

7. The Projective $\bar{\partial}$-Problem

8. Curves in $\mathbb{P}^{2}$

9. Curves in $\mathbb{P}^{n}$

10. Boundaries of Higher Dimension

11. The General Result in Terms of Moment Conditions

12. The Relation to Dolbeault-Henkin

\footnotetext{
${ }^{1}$ Research partially supported by the NSF
} 
$\S 1$. Introduction. Let $M \subset X$ be a compact oriented submanifold of dimension $2 p-1$ in a quasi-projective manifold $X$. The question addressed here is: under what conditions does $M$ bound a complex submanifold of dimension $p$, or more generally, a holomorphic $p$-chain in $X$ ?

When $X$ is Stein, much is known. For example if $p>1$, a necessary and sufficient condition is that $M$ be maximally complex, i.e., that $\operatorname{dim}_{\mathbb{C}}\left(T_{x} M \cap i T_{x} M\right)=p-1$ for all $x \in M$ (see $\left.\left[\mathrm{HL}_{1}\right]\right)$. This represents a geometric generalization of the BochnerHartogs extension theorem for CR functions [B]. When $p=1$, a necessary and sufficient condition is that $\int_{M} \omega=0$ for all holomorphic 1-forms $\omega \in \Omega^{1}\left(\mathbb{C}^{n}\right)$ (see $\left.\left[\mathrm{HL}_{1}\right]\right)$. This is called the moment condition.

For a connected curve $\gamma$ in $\mathbb{C}^{n}$ there is another, quite different characterization: $\gamma$ bounds a 1-dimensional analytic subvariety in $\mathbb{C}^{n}$ if and only if it is not polynomially convex (i.e., the polynomials are not uniformly dense in $C(\gamma)$ ). When this occurs, the subvariety with boundary $\gamma$ coincides with the polynomial hull of $\gamma$. This work goes back to classical results of J. Wermer $\left[\mathrm{W}_{1}\right]$ for smooth curves with generalizations to progressively less regular curves by E. Bishop, H. Royden, G. Stolzenberg, H. Alexander and M. Lawrence (see [L]).

In $\mathbb{C}^{n}$ there is yet another characterization of boundaries of holomorphic chains in terms of the positivity of linking numbers due to Alexander and Wermer $[\mathrm{A}]$, $\left[\mathrm{AW}_{2}\right],\left[\mathrm{W}_{2}\right]$.

For $X=\mathbb{P}^{n}-\mathbb{P}^{n-k}, k>1$ there are results in $\left[\mathrm{HL}_{2}\right]$ which extend those of $\left[\mathrm{HL}_{1}\right]$. When $p>k$, maximal complexity suffices, and when $p=k$ there is a moment condition which is necessary and sufficient. (See the discussion in §2.)

However, the case where $p<k$, which corresponds essentially to taking $X=\mathbb{P}^{n}$, is quite difficult. There is beautiful work of Dolbeault and Henkin $\left[\mathrm{DH}_{1,2}\right]$ which recasts the problem in terms of certain structure in a Cauchy-Radon tranform of $M$. Nevertheless, results for boundaries in $\mathbb{P}^{n}$ similar to those outlined above have not been established. This is the first in a series of articles devoted to that problem. In general terms what we prove here is the following.

The Main Result.Consider a maximally complex submanifold $M$ of dimension $2 p-1$ in $\mathbb{P}^{n}$. Fix a projection $\pi: \mathbb{P}^{n}--->\mathbb{P}^{p}$ which is proper on $M$, and a "base point" $a \in \mathbb{P}^{p}-\pi(M)$. Then $M$ bounds a holomorphic p-chain which is positive and $\ell$-sheeted over $a$ if and only if $M$ satisfies a sequence of (explicit and algorithmically computable) non-linear moment conditions which depend on $\ell$.

This generalizes the results in $\left[\mathrm{HL}_{1,2}\right]$ where $\ell=0$, and complements those in $\left[\mathrm{DH}_{1,2}\right]$. An expanded discussion of the main result, including a detailed exposition of the moment conditions, is given in $\S 3$. These moment conditions involve certain universal Newton polynomials. They constitute a complete set of computable obstructions to solving the central problem. The main theorem and its proof appear in $\S \S 8-11$.

In their paper $\left[\mathrm{DH}_{1,2}\right]$ Dolbeault and Henkin also fix a projection $\pi$ as above and consider certain naturally associated Cauchy transforms $T_{*}(M, \pi)$. They then vary the projection in a neighborhood $U$ of $\pi$ on the Grassmannian. Their result asserts that $M$ bounds a holomorphic $p$-chain iff second derivatives of $T_{*}(M, \pi)$ can be written as second derivatives of a linear combination of solutions of the "shock equation" on $U$. This result is quite general, but it leaves open the difficult problem 
of determining, for a given boundary $M$, whether $T_{*}(M, \pi)$ can be written in such a fashion.

One of the major difficulties in establishing $\mathbb{P}^{n}$-versions of this problem is the lack of uniqueness of the solution. If $d T=M$, then $d(T+C)=M$ for any algebraic $p$-cycle $C$ in $\mathbb{P}^{n}$. Our condition of "positivity with $\ell$ sheets over a point" vastly reduces the ambuguity in the solution and enables the derivation of the moment conditions. The Dolbeault-Henkin theorem is much less explicit but automatically incorporates the full order of non-uniqueness.

In pondering this problem one naturally wonders if boundaries of varieties in $\mathbb{P}^{n}$ can be characterized by the vanishing of the integrals of certain forms with singularities. For curves this leads to the following question. Given a compact irreducible complex analytic curve $C \subset \mathbb{P}^{n}$ with boundary $d C \neq \emptyset$, does there exist a divisor $D$ in $\mathbb{P}^{n}$ such that $D \cap C=\emptyset$ ? If this were always true then a given real curve $\gamma \subset \mathbb{P}^{n}$ would bound an analytic variety iff it satisfied the standard moment condition in some affine chart. This would amount to the condition that $\int_{\gamma} \omega=0$ for all rational 1-forms with poles on a fixed divisor $D$. However, in beautiful work which employs the Rosenlicht generalized jacobians for singular curves, Bruno Fabré $\left[\mathrm{Fa}_{*}\right]$ has shown that this is not the case. There exist many such varieties $C \subset \mathbb{P}^{2}$ (proper subsets of algebraic curves!) which have the property that they meet every divisor.

In considering this problem it is also natural to ask if there exist extensions of the work of Wermer and others from the affine to the projective case. The answer is yes. In $\left[\mathrm{HL}_{3}\right]$ we introduce the concept of the projective hull $\widehat{\gamma}$ of a subset $\gamma \subset \mathbb{P}^{n}$. When $\gamma$ is a smooth curve which bounds an analytic variety $C \subset \mathbb{P}^{n}$, one has $C \subset \widehat{\gamma}$ and there is much evidence for the conjecture that at least for real analytic $\gamma$ one has

$$
\widehat{\gamma}= \begin{cases}\Sigma & \text { when } C \text { is a subset of an irreducible algebraic curve } \Sigma \\ C & \text { otherwise. }\end{cases}
$$

In fact it has been shown in complete generality that for any compact subset $K \subset \mathbb{P}^{n}$, the set $\widehat{K}^{-}-K$ is 1-concave in the sense of [DL]. In particular, on any domain in $\mathbb{P}^{n}-K$ where the Hausdorff 2-measure of $\widehat{K}^{-}-K$ is finite, $\widehat{K}^{-}-K$ is a 1-dimensional analytic subvariety. Furthermore, for any finite union of smooth pluripolar curves (e.g., real analytic curves) $\gamma \subset \mathbb{P}^{2}$, the Hausdorff dimension of $\widehat{\gamma}$ is $\leq 2$. These results $\left(\left[\mathrm{HL}_{3}\right]\right)$ make plausible certain specific analogues of the work of Alexander and Wermer $\left[\mathrm{AW}_{2}\right],\left[\mathrm{W}_{2}\right]$ for submanifolds of projective space. (See $\left.\left[\mathrm{HL}_{4}\right].\right)$

Recall that the polynomial hull of a set $Y \subset \mathbb{C}^{n}$ gives a concrete realization of the Gelfand spectrum of the Banach algebra obtained by taking the uniform closure of the the polynomials in $C(Y)$ (cf. [Ho] or $\left.\left[\mathrm{AW}_{1}\right]\right)$. Interestingly there exists a projective Gelfand transformation defined for graded Banach algebras, which for subsets of $\mathbb{P}^{n}$ gives a concrete realization of the projective hull mentioned above. It is related to the usual Gelfand Transform much as Grothendieck's Proj is related to the spectrum of a ring. All this is introduced and discussed in $\left[\mathrm{HL}_{3}\right]$.

A special but quite interesting instance of the problem considered here is that of characterizing the boundary values of meromophic mappings $F: \Omega \rightarrow X$ where $\Omega$ 
is a domain in $\mathbb{C}^{p}$ and $X$ is a projective variety. If $f: d \Omega \rightarrow X$ is a given function, consider its graph $M=\operatorname{graph}(f) \subset \Omega \times X \subset \mathbb{P}^{p} \times \mathbb{P}^{N} \subset \mathbb{P}^{p N+p+N}$ and look for a variety $V$ with $d V=M$. Any such $V$, if it exists, will lie in $\mathbb{P}^{p} \times X$ but in general it will not be the graph of a function over $\Omega$. Nevertheless, there is a relatively simple solution to this problem which is presented in $\left[\mathrm{HL}_{4}\right]$.

\section{$\S 2$. Statement of the Problem.}

Suppose that $M$ is a compact oriented submanifold of class $C^{1}$ and dimension $2 p-1 \geq 1$ in complex projective $n$-space $\mathbb{P}^{n}$. This paper addresses the following:

Main Problem. Under what conditions on $M$ does there exist a holomorphic $p$-chain in $\mathbb{P}^{n}$ with boundary $M$ ? That is, under what conditions does there exist a holomorphic $p$-chain $T$ in $\mathbb{P}^{n}-M$ such that, as a current in $\mathbb{P}^{n}, T$ has finite mass and satisfies $d T=M$ ?

Recall that a holomorphic $p$-chain in a complex manifold $Y$ is a current $T$ of dimension $2 p$ which can be written as a locally finite sum

$$
T=\sum_{j} n_{j}\left[V_{j}\right]
$$

where for each $j, n_{j} \in \mathbb{Z}$ and $\left[V_{j}\right]$ is the current given by integration over (the regular points of) a canonically oriented irreducible analytic subvariety of dimension $p$ in $Y$.

There are two fundamental issues which we shall not discuss here since they are well covered in the literature.

Boundary Regularity. It is shown in $\left[\mathrm{HL}_{1}\right],[\mathrm{H}]$ that if $d T=M$ as above, then one has boundary regularity almost everywhere in the following sense. There exists a compact subset $\Sigma \subset M$ of $(2 p-1)$-dimensional measure 0 , such that if $U \subset M-\Sigma$ is an open set where $M$ is of class $C^{k}, 1 \leq k \leq \omega$, then there is an open set $\widetilde{U} \subset X$ with $\widetilde{U} \cap M=U$ in which $T$ is a complex manifold with $C^{k}$-boundary $M$.

Allowable Singularities on $M$. The regularity conditions on $M$ can be weakened to allow "scar sets". (See $[\mathrm{H}]$ for a complete discussion.) The solutions we shall discuss here will also exist for these more general boundaries, however we will restrict attention to the smooth case.

Some History. Whenever the submanifold $M$ lies in the complement of a linear subspace of codimension $\leq p$, our problem is well understood. To state the results we need two basic concepts.

Definition 2.1. The submanifold $M$ is said to be maximally complex if for each $a \in M$,

$$
\operatorname{dim}_{\mathbb{C}}\left(T_{a} M \cap i T_{a} M\right)=p-1 .
$$

This is equivalent to the condition that

$$
M=M_{p-1, p}+M_{p, p-1}
$$

where $M=\sum_{r+2=2 p-1} M_{r, s}$ is the Dolbeault decomposition of the current $M$. That is, the Dolbeault components $M_{r, s}=0$ for $|r-s|>1$. This condition is automatic when $p=1$. 
Definition 2.2. The submanifold $M$ is said to satisfy the moment condition on an open neighborhood $\Omega \subset \mathbb{P}^{n}$ if

$$
\int_{M} \omega=0 \quad \text { for all } \omega \in \mathcal{E}^{p, p-1}(\Omega) \text { with } \bar{\partial} \omega=0 \text {. }
$$

Definition 2.3. Let $M$ be a $2 p$-1-dimensional current with compact support in a complex manifold $\Omega$. Then $M$ is said to bound a holomorphic $p$-chain in $\Omega$ if there exists a holomorphic $p$-chain $T$ with finite mass in $\Omega-\operatorname{supp}(M)$ and supp $(T) \subset \subset \Omega$ with the property that $d T=M$ as currents in $\Omega$. By [HS] any such $T$ is unique modulo holomorphic chains with compact support in $\Omega$

Theorem 2.4. $\left[\mathbf{H L}_{1,2}\right]$. Let $M \subset \mathbb{P}^{n}$ be a compact oriented $(2 p-1)$-dimensional submanifold of class $C^{1}$, and suppose $M$ is contained in

$$
\Omega=\mathbb{P}^{n}-\mathbb{P}^{n-k}
$$

where $\mathbb{P}^{n-k}$ is some linear subspace of dimension $n-k$. Then $M$ is the boundary of a holomorphic $p$-chain in $\Omega$ if either

(1) $k<p$ and $M$ is maximally complex, or

(2) $\quad k=p$ and $M$ is maximally complex and satisfies the moment condition in $\Omega$

Note that when $k=1, \Omega \cong \mathbb{C}^{n}$ and the moment condition enters only for curves $(p=1)$. Maximally complex submanifolds of higher dimension in $\mathbb{C}^{n}$ automatically bound holomorphic chains. As noted in $\S 1$, the case of curves in $\mathbb{C}^{n}$ is alaso related to the study of polynomial hulls and has a long history going back to fundamental work of Wermer.

Note 2.5. If $M$ bounds a holomorphic chain in $\mathbb{P}^{n}$, then $M$ bounds a holomorphic chain in $\Omega=\mathbb{P}^{n}-\mathbb{P}^{n-(p+1)}$ for almost all linear subspaces $\mathbb{P}^{n-(p+1)}$. Hence the cases $k>p$ not considered in Theorem 2.4 are essentially equivalent to the one being studied here.

In projective space maximal complexity does not guarantee that $M$ bounds a holomorphic chain, even for $p>>1$. Consider the following examples.

Example 2.6. Let $M$ be a smooth real hypersurface in a complex $p$-dimensional submanifold $X^{p} \subset \mathbb{P}^{n}$, and assume that $M$ is not homologous to 0 in $X^{p}$. Then $M$ cannot bound a holomorphic chain in $\mathbb{P}^{n}$ for any such chain would necessarily have support in $X^{p}$.

Example 2.7. Let $Y \subset \mathbb{P}^{m}$ be any projective variety and let $C \subset \mathbb{C}^{2}$ be the graph of the function $w=\exp \left(z+\frac{1}{z}\right)$. Let $M$ be any real hypersurface in $Y \times C$ which is homologous to $Y \times \gamma$ where $\gamma \subset C$ is the curve given by retricting the graph to $|z|=1$. Embed $M$ into projective space by the composition $M \subset Y \times C \subset$ $\mathbb{P}^{m} \times \mathbb{C}^{2} \subset \mathbb{P}^{m} \times \mathbb{P}^{2} \subset \mathbb{P}^{3 m+2}$ where the last map is the Segré embedding. Again $M$ cannot bound a holomorphic chain in $\mathbb{P}^{3 m+2}$ for any such chain would necessarily have support in $Y \times C$. 
The Non-uniqueness in the Projective Case. A subtle difficulty of our main problem is the non-uniqueness of solutions when they exist. If $T$ is a holomorphic chain with boundary $M$ in $\mathbb{P}^{n}$, then so is $T+T_{0}$, where $T_{0}$ is any holomorphic chain with $d T_{0}=0$, i.e., any algebraic cycle.

There are of course canonical solutions. When $M$ is connected, there is a unique solution that is irreducible in $\mathbb{P}^{n}-M$. More generally there is a unique solution of least mass. However, these "simpliest" solutions are hard to characterize with a process that also detects solutions for complicated, highly non-connected boundaries.

Structuring the Problem. To make the general problem more tractible one could narrow the focus of the question. Two natural ways of doing this are as follows.

1. Prescribe the homology class of the solution. We fix a homology class $u \in H_{2 p}\left(\mathbb{P}^{n}, M ; \mathbb{Z}\right)$ such that $\delta u=[M]$ where $\delta: H_{2 p}\left(\mathbb{P}^{n}, M ; \mathbb{Z}\right) \rightarrow H_{2 p-1}(M ; \mathbb{Z})$ is the boundary map, and search for a holomorphic chain in $u$. There is a short exact sequence

$$
0 \longrightarrow H_{2 p}\left(\mathbb{P}^{n} ; \mathbb{Z}\right) \longrightarrow H_{2 p}\left(\mathbb{P}^{n}, M ; \mathbb{Z}\right) \stackrel{\delta}{\rightarrow} H_{2 p-1}(M ; \mathbb{Z}) \longrightarrow 0
$$

from which we conclude the following.

Lemma 2.8. A class $u \in H_{2 p}\left(\mathbb{P}^{n}, M ; \mathbb{Z}\right)$ with $\delta u=[M]$ is completely determined by its intersection number $u \cdot \mathbb{P}^{n-p}$ with any linear subspace $\mathbb{P}^{n-p} \subset \mathbb{P}^{n}-M$.

As noted in 2.5 our problem is equivalent to looking for solutions in open subsets of the form $\Omega=\mathbb{P}^{n}-\mathbb{P}^{n-p-1}$ which contain $M$. The homomorphism $H_{2 p-1}(\Omega, M ; \mathbb{Z}) \rightarrow H_{2 p-1}\left(\mathbb{P}^{n}, M ; \mathbb{Z}\right)$ induced by inclusion, is an isomorphism by general position arguments. Under the linear projection

$$
\pi: \mathbb{P}^{n}-\mathbb{P}^{n-p-1} \longrightarrow \mathbb{P}^{p}
$$

the class $\pi_{*} u$ corresponds to an integer-valued function $C_{0}$ with $d C_{0}=d \pi_{*} u=\pi_{*} M$. In particular,

$$
C_{0}(a)=u \cdot \mathbb{P}_{a}^{n-p} \quad \text { where } \quad \mathbb{P}_{a}^{n-p} \equiv \pi^{-1}(a)
$$

for any $a \in \mathbb{P}^{p}-\pi M$.

Now integer-valued functions $C_{0}$ with $d C_{0}=\pi_{*} M$ are determined up to an integer constant. Thus by Lemma 2.8 we see that:

Prescribing the homology class $u$ is equivalent to choosing such a function $C_{0}$ for some projection $\pi$.

2. Require the solution $T$ to be positive. We could restrict the question by asking only for positive holomorphic chains: $T=\sum_{i} n_{i}\left[V_{i}\right]$ with $n_{i} \in \mathbb{Z}^{+}$, having $d T=M$. This approach has several features:

- It covers the case where $M$ is connected, which is already of considerable interest. 
- If one also prescribes the homology class of the solution, then there exists at most a finite-dimensional space of solutions.

- The integer-valued function $C_{0}$ discussed above becomes the sheeting number of $T$ over $\mathbb{P}^{p}$ for the projection $\pi$.

- Positive solutions in a homology class $u$ are exactly the solutions to the Plateau Problem for $M$ in $u$ for any Kaehler metric on $\mathbb{P}^{n}$.

We shall impose somewhat weaker restrictions.

Our Approach: Prescribe the homology class of the solution and require positivity only over one point for some projection $\pi$. We shall find that this approach

- covers the case where $M$ is connected,

- still guarantees finite-dimensionality of the solution space,

- recaptures Theorem 2.4 as a special case, and

- leads to explicit non-linear moment conditions necessary and sufficient for the solution.

In fact when solutions to this problem exist, one also captures the canonical solution (of smallest mass) by minimizing the sheeting number over the given point.

\section{$\S 3$. Discussion of the Main Result.}

Before launching a complete exposition we present the main ideas in outline form. For clarity of exposition we focus here on the hypersurface case. However, the result in general codimensions is very much the same (see $\S \S 9$ and 10).

Fix a compact oriented $C^{1}$-submanifold $M \subset \mathbb{P}^{n+1}$ of dimension $2 n-1 \geq 1$ which is maximally complex. Fix a point $\mathbb{P}^{0} \notin M$. Choose homogeneous coordinates $\left[z_{0}: \cdots: z_{n+1}\right]$ for $\mathbb{P}^{n+1}$ with $\mathbb{P}^{0}=[0: \cdots: 0: 1]$ and let

$$
\Omega \equiv \mathbb{P}^{n+1}-\mathbb{P}^{0} \stackrel{\pi}{\rightarrow} \mathbb{P}^{n}
$$

be the projection defined by $\pi\left(\left[z_{0}: \cdots: z_{n+1}\right]\right)=\left[z_{0}: \cdots: z_{n}\right]$. Let $w$ be the tautological cross-section of $\left.\pi^{*} \mathcal{O}_{\mathbb{P}^{n}}(1) \cong \mathcal{O}_{\mathbb{P}^{n+1}}(1)\right|_{\Omega}$. Then each push-forward $\pi_{*}\left(w^{d} M\right)$ with $d \geq 0$ is a well-defined current of degree one with values in the bundle $\mathcal{O}_{\mathbb{P}^{n}}(d)$. Let $\pi_{*}\left(w^{d} M\right)^{0,1}$ denote the Dolbeault component of bidegree $(0,1)$ of this current. Then the maximal complexity of $M$ (cf. (2.1)) and the fact that $d M=0$ imply that

$$
\bar{\partial} \pi_{*}\left(w^{d} M\right)^{0,1}=0 \quad \text { for } d=0,1,2, \ldots
$$

Now fix a point $a \in \mathbb{P}^{n}-\pi(M)$. Then standard arguments show that there exists a unique generalized section $C_{d}^{o}$ of $\mathcal{O}_{\mathbb{P}^{n}}(d)$ such that

$$
\bar{\partial} C_{d}^{o}=\pi_{*}\left(w^{d} M\right)^{0,1}
$$

$C_{d}^{o}$ vanishes to order $d$ at $a$, 
and any solution of (3.1) differs from $C_{d}^{o}$ by a global holomorphic section of $\mathcal{O}_{\mathbb{P}^{n}}(d)$, i.e., a homogeneous polynomial of degree $d$ in $\left(z_{0}, \ldots, z_{n}\right)$.

The main idea now is the following. Suppose there exists a holomorphic chain $T$ with $d T=M$ which misses $\mathbb{P}^{0}$. Then $C_{d} \equiv \pi_{*}\left(w^{d} T\right)$ is a solution of (3.1) and therefore can be written in the form

$$
C_{d} \equiv \pi_{*}\left(w^{d} T\right)=C_{d}^{o}+p_{d}
$$

for a unique homogenous polynomial

$$
p_{d} \in H^{0}\left(\mathbb{P}^{n}, \mathcal{O}(d)\right) \cong \mathbb{C}\left[z_{0}, \ldots, z_{n}\right]_{d} \equiv \mathcal{H}(d) .
$$

Let us suppose further that $T$ is positive over $a$, i.e., positive in $\pi^{-1}(U)$ for some neighborhood $U$ of $a$ in $\mathbb{P}^{n}-\pi(M)$. Then $\left.C_{0}\right|_{U} \equiv \ell \in \mathbb{Z}^{+}$is the sheeting number of $T$ over $U$, and with respect to any non-vanishing section $\sigma$ of $\mathcal{O}_{U}(1)$, there are functions $f_{1}, \ldots, f_{\ell}$ (locally defined and holomorphic outside a divisor, as in the Weierstrauss Preparation Theorem) such that

$$
\frac{C_{d}}{\sigma^{d}}=f_{1}^{d}+f_{2}^{d}+\cdots+f_{\ell}^{d} .
$$

A sequence $\left\{C_{d}\right\}_{d=0}^{\infty}$ satisying (3.3) for some, and therefore any, $\sigma$ is called a Newton hierarchy of level $\ell$. Such hierarchies are freely determined by the first $\ell+1$ functions $C_{0}, \ldots, C_{\ell}$. The remaining functions can be written recursively by explicit universal homogeneous polynomial expressions

$$
C_{d}=Q_{d, \ell}\left(C_{1}, \ldots, C_{\ell}\right) \quad \text { for } d>\ell .
$$

(When $\ell=0$, then $Q_{d, \ell}=0$ for all $d$.) If these identities are satisfied then there exist holomorphic sections $f_{1} \sigma, \ldots, f_{\ell} \sigma$ such that (3.3) holds. This leads to the following.

Theorem 3.1. (The Main Result for Hypersurfaces). There exists a holomorphic chain $T$ with $d T=M$ which is supported in $\mathbb{P}^{n+1}-\mathbb{P}^{0}$ and is positive over a point $a \in \mathbb{P}^{p}-\pi(M)$ if and only if there exist an integer $\ell \geq 0$ and homogeneous polynomials $p_{d} \in \mathcal{H}(d)$ for $d=1, \ldots, \ell$ such that in some neighborhood of $a$

$$
C_{k}^{o} \equiv Q_{k, \ell}\left(C_{1}, \ldots, C_{\ell}\right) \quad \bmod \mathcal{H}(k) \quad \text { for all } k>\ell .
$$

where $C_{d}=C_{d}^{o}+p_{d}$ for $d=1, \ldots, \ell$.

Condition (3.5) constitutes a non-linear moment condition. The essential point is that the coefficients of degree $>k$ in the power series expansion of $C_{k}$ at $a$ can be written, via the Bochner-Martinelli kenel, as certain explicit integrals over $M$. Condition (3.5) says that these coefficients for $k>\ell$ must equal certain polynomial expressions in the coefficients of $C_{1}, \ldots, C_{\ell}$ and $p_{1}, . ., p_{\ell}$.

The polynomials $p_{1}, \ldots, p_{\ell}$ are initially free. However, in general the equations (3.5) will determine a certain number them. When we have an $\ell=\ell_{0}$ so that $T$ exists and has no boundary-free components, then all of $p_{1}, . ., p_{\ell_{0}}$ will be determined. However, if we then increase $\ell$, solutions will continue to exist but there will remain some freedom in choosing $p_{1}, \ldots, p_{\ell}$. This corresponds to the freedom in adding positive boundary-free components to the original solution.

All this is best illustrated by considering the special case of curves in $\mathbb{P}^{2}$ (which is essentially as complicated as the general case). 
Example 3.2. (Curves in $\mathbb{P}^{2}$ ). Let $\gamma \subset \mathbb{P}^{2}$ be a compact oriented $C^{1}$-curve without boundary and not necessarily connected. Fix a point $\mathbb{P}^{0} \in \mathbb{P}^{2}$ not on $\gamma$. Choose homogeneous coordinates $\left[z_{0}: z_{1}: z_{2}\right]$ so that $\mathbb{P}_{0}=[0: 0: 1]$ and define $\pi: \mathbb{P}^{2}-\mathbb{P}^{0} \rightarrow \mathbb{P}^{1}$ by setting $\pi\left(\left[z_{0}: z_{1}: z_{2}\right]\right)=\left[z_{0}: z_{1}\right]$ as above. Suppose $a \equiv[1: 0] \notin \pi(\gamma)$. Consider the affine coordinate $z=z_{1} / z_{0}$ on the open subset $U=\left\{z_{0} \neq 0\right\} \subset \mathbb{P}^{1}$. Then $w\left(\left[z_{0}: z_{1}: z_{2}\right]\right)=z_{2}$ is the tautological section of $\pi^{*} \mathcal{O}(1)$ on $\mathbb{P}^{2}-\mathbb{P}^{0}$, and the integral

$$
C_{d}(z)=\frac{1}{2 \pi i} \int_{\gamma} \frac{w^{d}}{\zeta-z} d \zeta
$$

solves the equation ${ }^{2} \bar{\partial} C_{d}=\pi_{*}\left(w^{d} \gamma\right)^{0,1}$.

Expanding (3.6) in a neighborhood of 0 we find that

$$
C_{d}(z)=\sum_{k=0}^{\infty}\left\{\frac{1}{2 \pi i} \int_{\gamma} \frac{w^{d}}{\zeta^{k+1}} d \zeta\right\} z^{k} \equiv \sum_{k=0}^{\infty} A_{k}(d) z^{k}
$$

and so

$$
C_{d}^{o}(z)=\sum_{k=d+1}^{\infty} A_{k}(d) z^{k}
$$

where

$$
A_{k}(d)=\frac{1}{2 \pi i} \int_{\gamma} \frac{w^{d}}{\zeta^{k+1}} d \zeta
$$

are classical moments of the curve $\gamma$.

We now have free polynomials $p_{d}(z)=c_{0}(d)+c_{1}(d) z+\cdots+c_{d}(d) z^{d}$ for $d=1, \ldots, \ell$. Plugging into the equations (3.5) gives a sequence of polynomial relations in the finite set $\left\{c_{k}(d)\right\}$ and the moments $\left\{A_{k}(d)\right\}_{k>d>\ell}$.

Consider for example the special case $\ell=1$. Then there is only one free polynomial

$$
p_{1}(z)=c_{0}+c_{1} z
$$

which means two free constants to determine. In this case the equations (3.5) have the particularly simple form

$$
C_{d}(z) \equiv\left(C_{1}^{o}(z)\right)^{d} \equiv\left(c_{0}+c_{1} z+C_{1}(z)\right)^{d} \quad \bmod \mathcal{H}(d)
$$

for $d \geq 2$. The first two non-zero coefficients of $C_{2}$ give

$$
A_{3}(2)=2 A_{3}(1) c_{0}+2 A_{2}(1) c_{1} \quad \text { and } \quad A_{4}(2)=2 A_{4}(1) c_{0}+2 A_{3}(1) c_{1}+A_{2}(1)^{2}
$$

Assume $A_{3}(1)^{2}-A_{2}(1) A_{4}(1) \neq 0$ and for notational convenience let $c_{k}=A_{k}(1)$ for $k=0,1$ denote the solutions of the equations above. Then we have the following.

\footnotetext{
${ }^{2}$ Note that this can be thought of as a scalar equation by writing

$$
C_{d}(z)=\left\{\frac{\tau_{d}(z)}{z_{0}^{d}}\right\} z_{0}^{d}=\left\{\frac{1}{2 \pi i} \int_{\gamma} \frac{\left(w / z_{0}\right)^{d}}{\zeta-x} d \zeta\right\} z_{0}^{d}
$$
}

with respect to the cross-section $\sigma=z_{0}$ of $\mathcal{O}_{\mathbb{P}^{1}}(1)$ and then setting $z_{0}=1$. 
Corollary 3.3. Necessary and sufficient conditions that $\gamma$ bound a holomorphic 1-chain which is positive and 1-sheeted over 0 is that the non-linear moment conditions

$$
A_{k}(d)=\sum_{j_{1}+\cdots+j_{r}=k} c_{d, J} A_{j_{1}}(1) \cdots A_{j_{r}}(1)
$$

hold for all $k>d>2$ where $c_{d, J}$ are the obvious combinatorial constants. Alternatively, in recurssive form this can be written as

$$
A_{k}(d)=\sum_{i+j=k} A_{i}(d-1) A_{j}(1)
$$

for all $k>d \geq 2$.

Note 3.4. The case $\ell=0$ corresponds to the existence of a solution with compact support in $\mathbb{C}^{2}=\mathbb{P}^{2}-\overline{\pi^{-1}(a)}$. Here our moment conditions reduce to the linear moment conditions $A_{k}(d)=0$ for all $k>d>0$. Making the substitution $z=1 / \zeta$ in (3.7) we recover exactly the moment condition of $\left[\mathrm{HL}_{1}\right]$.

\section{$\S 4$. Newton Hierarchies}

The following material is classical but central to our results. For the convenience of the reader we review this material in a form that is particularly adapted to our needs.

Definition 4.1. A sequence of complex numbers $\left\{c_{d}\right\}_{d=0}^{\infty}$ is called a Newton hierarchy of level $\ell$ if there exist complex numbers $b_{1}, \ldots, b_{\ell}$ such that

$$
c_{d}=b_{1}^{d}+\cdots+b_{\ell}^{d} \quad \text { for all } d \geq 0 .
$$

Note that $c_{0}=\ell$.

The main fact is that in a Newton hierarchy of level $\ell$ the numbers $c_{1}, \ldots, c_{\ell}$ can be freely prescribed and the remaining terms in the sequence are given by explicit polynomial expressions in terms of these.

Example 4.2. $(\ell=1) . \quad c_{d}=c_{1}^{d}$ for all $d$.

Example 4.3. $(\ell=2) . \quad c_{d}=b_{1}^{d}+b_{2}^{d}$ for $d=0,1,2$. Then $b_{1}$ and $b_{2}$ are the roots of the polynomial $x^{2}-\left(b_{1}+b_{2}\right) x+b_{1} b_{2}=x^{2}-c_{1} x+\frac{1}{2}\left(c_{1}^{2}-c_{2}\right)$. Thus, setting $S_{1}=c_{1}$ and $S_{2}=\frac{1}{2}\left(c_{1}^{2}-c_{2}\right)$ we see that $b_{j}^{d}-S_{1} b_{j}^{d-1}+S_{2} b_{j}^{d-2}=0$ for all $d \geq 2$. Consequently,

$$
c_{d}-S_{1} c_{d-1}+S_{2} c_{d-2}=0 \quad \text { for all } d>2 .
$$


Proposition 4.4. Let $\left\{S_{k}\left(c_{1}, \ldots, c_{k}\right)\right\}_{k=1}^{\infty}$ be the sequence of polynomials defined recursively by the equations

$$
c_{\ell}-S_{1} c_{\ell-1}+S_{2} c_{\ell-2}-\cdots+(-1)^{\ell} S_{\ell}=0 .
$$

Then setting

$$
\sigma_{k}\left(x_{1}, \ldots, x_{N}\right)=\sum_{i_{1}<\cdots<i_{k}} x_{i_{1}} \ldots x_{i_{k}} \quad \text { and } \quad c_{k}\left(x_{1}, \ldots, x_{N}\right)=\sum_{i=1}^{N} x_{i}^{k}
$$

we have

$$
\sigma_{k}(x)=S_{k}\left(c_{1}(x), \ldots, c_{k}(x)\right)
$$

in $\mathbb{C}\left[x_{1}, \ldots, x_{N}\right]$ for all $N \geq k$.

Proof. We first prove the assertion for $N=k$. This is easily checked for $k=1,2$. Assume inductively that it holds for all $k^{\prime}<k$. Note that

$$
\left(t-x_{1}\right) \ldots\left(t-x_{k}\right)=t^{k}-\sigma_{1}\left(x_{1}, \ldots, x_{k}\right) t^{k-1}+\cdots+(-1)^{k} \sigma_{k}\left(x_{1}, \ldots, x_{k}\right) .
$$

Substituting $t=x_{j}$ and summing over $j$ gives

$$
c_{k}(x)-\sigma_{1}(x) c_{k-1}(x)+\cdots+(-1)^{k} \sigma_{k}(x) k=0 .
$$

for $x=\left(x_{1}, \ldots, x_{k}\right)$. Therefore, by induction

$$
\begin{aligned}
\sigma_{k}(x)= & \frac{(-1)^{k+1}}{k}\left\{c_{k}(x)-\sigma_{1}(x) c_{k-1}(x)+\sigma_{2}(x) c_{k-2}(x)-\sigma_{3}(x) c_{k-3}(x)+\ldots\right\} \\
= & \frac{(-1)^{k+1}}{k}\left\{c_{k}(x)-S_{1}\left(c_{1}(x)\right) c_{k-1}(x)+S_{2}\left(c_{1}(x), c_{2}(x)\right) c_{k-2}(x)\right. \\
= & \left.-S_{3}\left(c_{1}(x), c_{2}(x), c_{3}(x)\right) c_{k-3}(x)+\ldots\right\}
\end{aligned}
$$

for $x=\left(x_{1}, \ldots, x_{k}\right)$. It remains only to establish this identity for $x=\left(x_{1}, \ldots, x_{N}\right)$ where $N>k$. For this we note that $P\left(x_{1}, \ldots, x_{N}\right) \equiv \sigma_{k}\left(x_{1}, \ldots, x_{N}\right)-S_{k}\left(c_{1}\left(x_{1}, \ldots, x_{N}\right), \ldots, c_{k}\left(x_{1}, \ldots, x_{N}\right)\right)$ is a symmetric homogeneous polynomial of degree $k$ such that $P\left(x_{1}, \ldots, x_{k}, 0, \ldots, 0\right)=$ 0 by the identity proved above. By symmetry it follows that $P\left(0, \ldots, 0, x_{i_{1}}, 0, \ldots, 0, x_{i_{k}}, 0, \ldots, 0\right)=$ 0 for all $1 \leq i_{1}<i_{2}<\cdots<i_{k} \leq N$. It follows that all the coefficients of $P$ are zero.

Corollary 4.5. The sequence $\left\{c_{d}\right\}_{d=0}^{\infty}$ is a Newton hierarchy of level $\ell$ if and only if $c_{0}=\ell$ and

$$
c_{k}-S_{1}\left(c_{1}\right) c_{k-1}+S_{2}\left(c_{1}, c_{2}\right) c_{k-2}-\cdots+(-1)^{\ell} S_{\ell}\left(c_{1}, \ldots, c_{\ell}\right) c_{k-\ell}=0
$$

for all $k>\ell$.

In other words, fix $\ell \geq 1$ and let $Q_{k, \ell}\left(c_{1}, \ldots, c_{\ell}\right)$ for $k>\ell$ be the sequence of polynomials generated recursively by setting $c_{j}=Q_{j, \ell}$ in equation (4.1). Then the sequence $\left\{c_{d}\right\}_{d=0}^{\infty}$ is a Newton hierarchy of level $\ell$ if and only if

$$
c_{k}=Q_{k, \ell}\left(c_{1}, \ldots, c_{\ell}\right) \quad \text { for all } k>\ell .
$$


Proof. Let $b_{1}, \ldots, b_{\ell}$ be the roots of the polynomial $p(t)=t^{\ell}-S_{1}\left(c_{1}\right) t^{\ell-1}+$ $S_{2}\left(c_{1}, c_{2}\right) t^{\ell-2}-\cdots+(-1)^{\ell} S_{\ell}\left(c_{1}, \ldots, c_{\ell}\right)$. Then $c_{d}=b_{1}^{d}+\cdots+b_{\ell}^{d}$ for $d=1, \ldots, \ell$ by Proposition 4.4. Substituting $t=b_{j}$ into $t^{k-\ell} p(t)$ and summing over $j$ completes the proof.

This gives the main result.

Theorem 4.6. Each $\ell$-tuple $\left(c_{1}, \ldots, c_{\ell}\right) \in \mathbb{C}^{\ell}$ extends to one and only one Newton hierarchy $\left\{c_{d}\right\}_{d=0}^{\infty}$ of level $\ell$. The numbers $c_{d}$ for $d>\ell$ are given by the universal polynomials (4.2).

Note 4.7. Let $\mathcal{N}_{\ell}$ denote the space of Newton Hierarchies of level $\ell$. Then $\mathcal{N} \equiv$ $\oplus_{d \geq 0} \mathcal{N}_{d}$ has a vector monoid structure. Given $\mathbf{C}=\left\{c_{d}\right\} \in \mathcal{N}_{\ell}, \widetilde{\mathbf{C}}=\left\{\widetilde{c}_{d}\right\} \in \mathcal{N}_{\tilde{\ell}}$, and $t \in \mathbb{C}$, we have

$$
t \cdot \mathbf{C}=\left\{t^{d} c_{d}\right\}_{d=0}^{\infty} \in \mathcal{N}_{\ell} \quad \text { and } \quad \mathbf{C}+\widetilde{\mathbf{C}}=\left\{c_{d}+\widetilde{c}_{d}\right\}_{d=0}^{\infty} \in \mathcal{N}_{\ell+\tilde{\ell}} .
$$

\section{$\S 5$. Newton Hierarchies with Coefficients in a Vector Bundle and their Associated Multisections.}

The notion of a Newton hierarchy extends immediately to powers of a line bundle. Let $\pi: E \rightarrow X$ be a holomorphic line bundle over a complex manifold $X$ of dimension $\mathrm{n}$, and suppose we are given holomorphic sections

$$
C_{d} \in H^{0}\left(X ; \mathcal{O}\left(E^{d}\right)\right) \quad \text { for each integer } d \geq 0
$$

where $E^{d}=E \otimes \cdots \otimes E$ (d-times). With respect to a local trivialization $\sigma \in$ $H^{0}(U ; \mathcal{O}(E))$ of $E$ over an open subset $U \subset X$ we can write

$$
C_{d}=c_{d} \sigma^{d} \quad \text { for all } d \geq 0 .
$$

where $c_{d} \in \mathcal{O}(U)$. If $\widetilde{\sigma} \in H^{0}(U ; \mathcal{O}(E))$ is another trivialization with $C_{d}=\widetilde{c}_{d} \widetilde{\sigma}^{d}$, then

$$
\widetilde{c}_{d}=\left(\frac{\sigma}{\widetilde{\sigma}}\right)^{d} c_{d} \quad \text { in } U
$$

Definition 5.1. A sequence of sections $\left\{C_{d}\right\}_{d=0}^{\infty}$ as in (5.1) with $C_{0} \equiv \ell \in \mathbb{N}$ is called a Newton Hierarchy of level $\ell$ with coefficients in $E$ if each point $x \in X$ has a neighborhood $U$ and a local trivialization $\sigma$ of $\left.E\right|_{U}$ such that the sequence of functions $\left\{C_{d} / \sigma^{d}\right\}_{d=0}^{\infty}$ is a Newton hierarchy of level $\ell$ at each point of $U$. By (5.2) if this condition holds for one trivialization over $U$, it holds for every trivialization.

Definition 5.2. By a multi-section of degree $\ell$ of a line bundle $\pi: E \rightarrow X$ we mean an effective divisor $D$ on the total space of $E$ such that $\left.\pi\right|_{D}$ is proper and of degree $\ell$.

Recall that by an effective divisor we mean a positive holomorphic n-chain on $E$ where $n=\operatorname{dim} X$, and by degree $\ell$ we mean that $\pi_{*} D=\ell[X]$ (or equivalently that the intersection 0 -cycle $D \bullet \pi^{-1}(x)$ (cf. [Fu]) has degree $\ell$ for all $x \in X$ ). The first main result of this section is the following. 
Theorem 5.3. Let $\pi: E \rightarrow X$ be a holomorphic line bundle over a complex manifold $X$. Then there is a one-to-one correspondence between Newton Hierarchies of level $\ell$ with coefficients in $E$ and multi-sections of $E$ of degree $\ell$.

This correspondence is given as follows. Let $w \in H^{0}\left(E, \mathcal{O}\left(\pi^{*} E\right)\right)$ denote the tautological cross-section $w(e)=e$. Then for a multi-section $D$ the associated Newton hierarchy is given by

$$
C_{d}=\pi_{*}\left(w^{d} D\right) \quad \text { for all } d \geq 0 .
$$

Proof. Since all notions are invariantly defined, it suffices to prove the result locally on $X$. Let $U \subset X$ be an open subset of $X$ and $\sigma$ a trivialization of $E$ over $U$. This gives an isomorphism

$$
U \times\left.\mathbb{C} \stackrel{\cong}{\rightrightarrows} E\right|_{U} \quad \text { defined by } \quad(x, t) \mapsto t \sigma(x) .
$$

In $U \times \mathbb{C}$ the tautological section $w$ has the form $w(t \sigma(x))=t \sigma(x)$ and so

$$
w / \sigma=t .
$$

Under (5.4) the restriction of $D$ to $\left.E\right|_{U}$ becomes an effective divisor in $U \times \mathbb{C}$ which is proper and of degree $\ell$ over $U$, which shall be denoted by $D_{U}$. Now it is classical that

$$
\operatorname{pr}_{*}\left(t^{d} D_{U}\right)=f_{1}^{d}+\cdots+f_{\ell}^{d}
$$

where pr: $U \times \mathbb{C} \rightarrow U$ is the projection, and $\left\{f_{1}(x), \ldots, f_{\ell}(x)\right\} \equiv \pi^{-1}(x) \bullet D_{U} \subset \mathbb{C}$ are the points of $D_{U}$ above $x \in U$ listed to multiplicity (See [H], [HL $\left.\mathrm{HL}_{1}\right]$ for example). It follows immediately that $c_{d} \equiv \operatorname{pr}_{*}\left(t^{d} D_{U}\right)$ is a Newton hierarchy of level $\ell$ and so $C_{d} \equiv c_{d} \sigma^{d}$ is a level- $\ell$ Newton hierarchy with coefficients in $E$.

For the converse suppose $\left\{C_{d}\right\}_{d=0}^{\infty}$ is a Newton hierarchy with coefficients in $E$, and write $C_{d}=c_{d} \sigma^{d}$ over $U$. Define holomorphic functions $s_{k} \in \mathcal{O}(U)$ by

$$
s_{k}(x)=S_{k}\left(c_{1}(x), \ldots, c_{\ell}(x)\right) \quad \text { for } \quad k=1, \ldots, \ell
$$

where the $S_{k}$ are the polynomials defined in Proposition 4.4. Define $P \in \mathcal{O}(U \times \mathbb{C})$ by

$$
P(x, t)=t^{\ell}-s_{1}(x) t^{\ell-1}+s_{1}(x) t^{\ell-1}-\cdots+(-1)^{\ell} s_{\ell}(x)
$$

and

$$
D_{U} \equiv \operatorname{Div}(P)=\frac{1}{2 \pi} d d^{C} \log |P| .
$$

This is a divisor in $U \times \mathbb{C}$ which is proper over $U$ and has the property that $\operatorname{pr}_{*}\left(t^{d} D_{U}\right)=c_{d}$ for all $d$. 
Note 5.5. One can check directly that the divisor of $P$ is invariantly defined. If $\widetilde{\sigma}$ is another trivialization of $E$ over $U$, and if we write $\widetilde{t}=(\sigma / \widetilde{\sigma}) t, \widetilde{c}_{d}=(\sigma / \widetilde{\sigma})^{d} c_{d}$ as above, then setting $\widetilde{s}_{k}=S_{k}\left(\widetilde{c}_{1}, \ldots, \widetilde{c}_{\ell}\right)$ we have $\widetilde{P}(x, \widetilde{t})=(\sigma / \widetilde{\sigma})^{\ell} P(x, t)$, which shows that $\operatorname{Div}(\widetilde{P})$ is the image of $\operatorname{Div}(\widetilde{P})$ under the change of trivialization.

We now pass to an important generalization of the discussion above. Consider the $q$-fold direct sum

$$
E^{\oplus q}=E \oplus \cdots \oplus E \stackrel{\Pi}{\rightarrow} X .
$$

For each $\lambda=\left(\lambda_{1}, \ldots, \lambda_{q}\right) \in \mathbb{C}^{q}$ consider the projection

$$
\pi_{\lambda}: E^{\oplus q} \longrightarrow E \quad \text { given by } \quad \pi_{\lambda}\left(e_{1}, \ldots, e_{q}\right)=\sum_{j=1}^{q} \lambda_{j} e_{j} .
$$

Note the commutative diagram

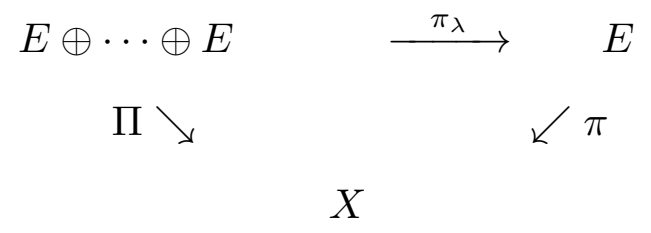

Let $w$ denote the tautological cross-section of $\pi^{*} E$ over $E$ as above and note that

$$
\pi_{\lambda}^{*} w=\sum_{j=1}^{q} \lambda_{j} w_{j} \equiv \lambda \cdot \mathbf{w}
$$

where $w_{j}$ denotes the $j^{\text {th }}$ tautological cross-section of $\Pi^{*} E \rightarrow E^{\oplus q}$, i.e., $w_{j}\left(e_{1}, \ldots, e_{q}\right)=$ $e_{j}$.

Suppose now that $D$ is a multi-section of $E^{\oplus q}$ of degree $\ell$, that is, a positive holomorphic $n$-chain on $E^{\oplus q}$ which is proper over $X$ and of degree $\ell$ (i.e., $\Pi_{*} D=$ $\ell[X])$. Then for each $\lambda, \pi_{\lambda}$ is proper on the support of $D$ and $D_{\lambda} \equiv\left(\pi_{\lambda}\right)_{*} D$ is a mult-section of $E$ of degree $\ell$. Hence there is an associated Newton hierarchy $\left\{\mathbf{C}_{d}(\lambda)\right\}_{d=0}^{\infty}$ of level $\ell$ on $X$ (with coefficients in $E$ ) defined by

$$
\mathbf{C}_{d}(\lambda)=\pi_{*}\left(w^{d} D_{\lambda}\right)=\Pi_{*}\left\{(\lambda \cdot \mathbf{w})^{d} D\right\} .
$$

Note that $\mathbf{C}_{d}(\lambda)$ is a homogeneous polynomial of degree $d$ in $\lambda$ with values in $H^{0}\left(X, \mathcal{O}\left(E^{d}\right)\right)$, i.e., $\mathbf{C}_{d} \in \operatorname{Sym}^{d}\left(\mathbb{C}^{q}\right)^{\vee} \otimes H^{0}\left(X, \mathcal{O}\left(E^{d}\right)\right)$. This polynomial can be re-expressed as

$$
\mathbf{C}_{d}(\lambda)=\sum_{|\alpha|=d}\left(\begin{array}{l}
d \\
\alpha
\end{array}\right) \lambda^{\alpha} \Pi_{*}\left\{w^{\alpha} D\right\}=\sum_{|\alpha|=d}\left(\begin{array}{l}
d \\
\alpha
\end{array}\right) C_{\alpha} \lambda^{\alpha}
$$

where $w^{\alpha}=w_{1}^{\alpha_{1}} \ldots w_{1}^{\alpha_{q}}$ and $|\alpha|=\alpha_{1}+\ldots \alpha_{q}$ as usual, and

$$
C_{\alpha} \equiv \Pi_{*}\left\{w^{\alpha} D\right\}
$$

is the $\alpha^{\text {th }}$ moment of $D$.

Conversely, suppose we are given elements $\mathbf{C}_{d} \in \operatorname{Sym}^{d}\left(\mathbb{C}^{q}\right)^{\vee} \otimes H^{0}\left(X, \mathcal{O}\left(E^{d}\right)\right)$ for $d \geq 0$, or equivalently, moments $C_{\alpha} \in H^{0}\left(X, \mathcal{O}\left(E^{|\alpha|}\right)\right.$ for $\alpha \geq(0, \ldots, 0)$. 
Definition 5.6. The family $\left\{C_{\alpha}\right\}_{\alpha \geq 0}$ is called a Newton family of level $\ell$ with coefficients in $E^{\oplus q}$ if the polynomials $\mathbf{C}_{d}(\lambda)=\sum_{|\alpha|=d}\left(\begin{array}{l}d \\ \alpha\end{array}\right) C_{\alpha} \lambda^{\alpha}$ satisfy the condition that

$$
\left\{\mathbf{C}_{d}(\lambda)\right\}_{d=0}^{\infty} \text { is a Newton hierarchy of level } \ell \text { for all } \lambda \in \mathbb{C}^{q} .
$$

It is straightforward to check from the result above and arguments in $\left[\mathrm{HL}_{1}, \S 7\right]$ that for any such family there exists a multi-section $D$ of degree $\ell$ of $E^{\oplus q}$ such that $C_{\alpha}=\Pi_{*}\left(w^{\alpha} D\right)$ for all $\alpha$. Hence we obtain the following.

Theorem 5.7. Let $\pi: E \rightarrow X$ be a holomorphic line bundle over a complex manifold $X$. Then there is a one-to-one correspondence between Newton families of level $\ell$ with coefficients in $E^{\oplus q}$ and multi-sections of $E^{\oplus q}$ of degree $\ell$.

This correspondence associates to a multi-section $D$ the family of moments $C_{\alpha}=$ $\Pi_{*}\left(w^{\alpha} D\right)$ where $w^{\alpha}=w_{1}^{\alpha_{1}} \ldots w_{q}^{\alpha_{q}}$ and $w_{j}$ is the $j^{\text {th }}$ tautological cross-section of $\Pi^{*} E^{\oplus q}$ defined above.

We now observe that Newton families $\left\{C_{\alpha}\right\}_{\alpha \geq 0}$ of level $\ell$ with coefficients in $E^{\oplus q}$ are universally determined by their terms of degree $\leq \ell$ as in (4.2). Indeed, by Corollary 4.5, our condition (5.10) above is equivalent to the condition that

$$
\mathbf{C}_{d}(\lambda)=Q_{d, \ell}\left(\mathbf{C}_{1}(\lambda), \ldots, \mathbf{C}_{\ell}(\lambda)\right) \quad \text { for all } d \geq \ell \text { and } \lambda \in \mathbb{C}^{q} .
$$

These universal polynomials $Q_{d, \ell}\left(\xi_{1}, \ldots, \xi_{\ell}\right)$ introduced in 4.5 are weighted homogeneous, that is,

$$
Q_{d, \ell}\left(t \xi_{1}, t^{2} \xi_{2}, \ldots, t^{\ell} \xi_{\ell}\right)=t^{d} Q_{d, \ell}\left(\xi_{1}, \ldots, \xi_{\ell}\right) \quad \text { for all } t \in \mathbb{C}
$$

or equivalently

$$
Q_{d, \ell}\left(\xi_{1}, \ldots, \xi_{\ell}\right)=\sum_{\beta_{1}+2 \beta_{2}+\cdots+\ell \beta_{\ell}=d} c_{\beta} \xi^{\beta}
$$

Let $e_{1}, \ldots, e_{q}$ denote the standard basis of $\mathbb{C}^{q}$ and write $C_{\beta} \equiv C_{\beta \cdot e} \equiv C_{\Sigma_{i} \beta_{i} e_{i}}$. One sees directly that

$$
Q_{d, \ell}\left(\sum_{i} C_{e_{i}} \lambda_{i}, \sum_{i j} C_{e_{i}+e_{j}} \lambda_{i} \lambda_{j}, \ldots, \sum_{|\beta|=\ell}\left(\begin{array}{c}
d \\
\beta
\end{array}\right) C_{\beta} \lambda^{\beta}\right)=\sum_{|\alpha|=d} Q_{\alpha, \ell}(C) \lambda^{\alpha}
$$

is a homogeneous polynomial of degree $d$ in $\lambda$ whose coefficients $Q_{\alpha, \ell}(C)$ are universal polynomials in the indeterminates $C=\left\{C_{\beta}\right\}_{|\beta| \leq \ell}$. This gives the following generalization of Corollary 4.5 to this case.

Proposition 5.8. Let $\left\{C_{\alpha}\right\}_{\alpha \geq 0}$ be a family of continuous sections of a complex line bundle $E$ on a space $X$. Then $\left\{C_{\alpha}\right\}_{\alpha \geq 0}$ is a Newton family of level $\ell$ with coefficients in $E^{\oplus q}$ iff

$$
C_{\alpha}=Q_{\alpha, \ell}(C) \quad \text { for all }|\alpha|>\ell
$$


where $C=\left\{C_{\beta}\right\}_{|\beta| \leq \ell}$.

$\S$. Vector Bundle-Valued Currents. In this section we prepare the general analytical tools for solving the main problem. In the next section we shall examine the concrete cases of interest.

For a smooth vector bundle $E$ over a manifold $X$ we denote by $\mathcal{E}_{p}^{\prime}(X, E)$ the space of $p$-dimensional currents of compact support on $X$ with values in $E$. By definition this is the topological dual of the space $\mathcal{E}^{p}\left(X, E^{*}\right)$ of smooth $p$-forms with values in $E^{*}$. To any smooth map $f: Y \rightarrow X$ between manifolds one associates a continuous map

$$
f_{*}: \mathcal{E}_{p}^{\prime}\left(Y, f^{*} E\right) \longrightarrow \mathcal{E}_{p}^{\prime}(X, E)
$$

given by $f_{*}(T)(\phi)=T\left(f^{*} \phi\right)$ for smooth forms $\phi \in \mathcal{E}^{p}\left(X, E^{*}\right)$

When $X$ and $Y$ are complex manifolds and $E$ and $f$ are holomorphic, there is a decomposition of currents $\mathcal{E}_{p}^{\prime}(X, E)=\oplus_{r+s=p} \mathcal{E}_{r, s}^{\prime}(X, E)$ preserved by $f_{*}$, and an operator

$$
\bar{\partial}: \mathcal{E}_{r, s}^{\prime}(X, E) \longrightarrow \mathcal{E}_{r, s-1}^{\prime}(X, E)
$$

which commutes with $f_{*}$. The space $\mathcal{E}_{r, s}^{\prime}(X, E)$ can be canonically identified with the space of compactly supported $(n-r, n-s)$-forms with distribution coefficients. Hence we shall write

$$
\mathcal{E}_{r, s}^{\prime}(X, E) \stackrel{\text { def }}{=} \mathcal{E}^{\prime n-r, n-s}(X, E)
$$

Under this identification $\bar{\partial}$ is identified with the usual operator and we have the following.

Theorem 6.1 (Dolbeault). There is a canonical isomorphism

$$
H^{p, q}\left(\mathcal{E}^{\prime *, *}(X, E)\right) \cong H_{\mathrm{cpt}}^{q}\left(X ; \Omega^{p} \otimes E\right)
$$

where the right hand side denotes sheaf cohomology with compact supports and coefficients in the sheaf of holomorphic $p$-forms with values in $E$.

$\S 7$. The Projective $\bar{\partial}$-Problem. Let $\mathbb{P}^{n}$ denote complex projective $n$-space with homogeneous coordinates $\left[z_{0}: \cdots: z_{n}\right]$, and let $\mathbb{P}^{n-p-1} \subset \mathbb{P}^{n}$ denote the linear subspace defined by $z_{0}=z_{1}=\cdots=z_{p}=0$. Consider the linear projection

$$
\Omega \stackrel{\text { def }}{=} \mathbb{P}^{n}-\mathbb{P}^{n-p-1} \stackrel{\pi}{\rightarrow} \mathbb{P}^{p}
$$

defined by $\pi\left(\left[z_{0}: \cdots: z_{n}\right]\right)=\left[z_{0}: \cdots: z_{p}\right]$. Note that $\pi: \Omega \rightarrow \mathbb{P}^{p}$ is isomorphic to the vector bundle $\mathcal{O}_{\mathbb{P}^{p}}(1)^{\oplus(n-p)}$.

Lemma 7.1. There is an isomorphism $\left.\pi^{*} \mathcal{O}_{\mathbb{P}^{p}}(1) \cong \mathcal{O}_{\mathbb{P}^{n}}(1)\right|_{\Omega}$.

Proof. This can be seen explicitly. Note that

$$
\mathcal{O}_{\mathbb{P}^{n}}(-1)=\left\{([z], \zeta) \in \mathbb{P}^{n} \times \mathbb{C}^{n+1}: \zeta=\lambda z \text { for some } \lambda \in \mathbb{C}\right\}
$$




$$
\pi^{*} \mathcal{O}_{\mathbb{P}^{p}}(-1)=\left\{([z], \zeta) \in \Omega \times \mathbb{C}^{p+1}: \zeta=\lambda\left(z_{0}, \ldots, z_{p}\right) \text { for some } \lambda \in \mathbb{C}\right\} .
$$

We define $\Psi:\left.\pi^{*} \mathcal{O}_{\mathbb{P}^{p}}(-1) \rightarrow \mathcal{O}_{\mathbb{P}^{n}}(-1)\right|_{\Omega}$ by

$$
\Psi\left(\left[z_{0}: \cdots: z_{n}\right],\left(\zeta_{0}, \ldots, \zeta_{p}\right)\right)=\left(\left[z_{0}: \cdots: z_{n}\right],\left(\zeta_{0}, \ldots, \zeta_{n}\right)\right)
$$

where for given $\left(z_{0}, \ldots, z_{n}\right)$ we have a unique $\lambda \in \mathbb{C}$ with $\zeta_{k}=\lambda z_{k}$ for $k=0, \ldots, p$ and we define $\zeta_{k}=\lambda z_{k}$ for $k>p$. The adjoint $\Psi^{*}$ gives the desired isomorphism.

Definition 7.2. The homogeneous coordinate functions $z_{0}, \ldots, z_{n}$ are holomorphic sections of $\mathcal{O}_{\mathbb{P}^{n}}(1)$. By the Lemma above each

$$
w_{k}=\left.z_{p+k}\right|_{Y}
$$

is a holomorphic section of $\left.\mathcal{O}_{\mathbb{P}^{n}}(1)\right|_{\Omega} \cong \pi^{*} \mathcal{O}_{\mathbb{P}^{p}}(1)$. The section $w_{k}$ will be called the $k^{\text {th }}$ tautological cross-section of $\pi^{*} \mathcal{O}_{\mathbb{P} p}(1)$.

It follows from Lemma 7.1 that there are isomorphisms $\left.\pi^{*} \mathcal{O}_{\mathbb{P}^{p}}(d) \cong \mathcal{O}_{\mathbb{P}^{n}}(d)\right|_{\Omega}$ for all integers $d$. Hence, by $\S 6$ the projection $\pi$ induces continuous homomorphisms on currents:

$$
\pi_{*}: \mathcal{E}_{*, *}^{\prime}\left(\Omega, \mathcal{O}_{\Omega}(d)\right) \longrightarrow \mathcal{E}_{*, *}^{\prime}\left(\mathbb{P}^{p}, \mathcal{O}_{\mathbb{P}}(d)\right)
$$

Proposition 7.3. Let $T \in \mathcal{E}_{p, p-1}^{\prime}(\Omega)$ be a $\bar{\partial}$-closed current on $\Omega$ and $\varphi \in H^{0}\left(\mathbb{P}^{n}, \mathcal{O}_{\mathbb{P}^{n}}(d)\right)$ a holomorphic section of $\mathcal{O}_{\mathbb{P}^{n}}(d)$ for $d \geq 0$. Then $\bar{\partial} \pi_{*}(\varphi T)=0$ and the associated $\bar{\partial}$-problem has solutions

$$
\left\{S \in \mathcal{E}^{\prime 0}\left(\mathbb{P}^{p}, \mathcal{O}_{\mathbb{P}^{p}}(d)\right): \bar{\partial} S=\pi_{*}(\varphi T)\right\} \cong H^{0}\left(\mathbb{P}^{p}, \mathcal{O}_{\mathbb{P}^{p}}(d)\right) .
$$

Proof. We have $\pi_{*}(\varphi T) \in \mathcal{E}_{p, p-1}^{\prime}\left(\mathbb{P}^{p}, \mathcal{O}_{\mathbb{P}^{p}}(d)\right) \cong \mathcal{E}^{\prime 0,1}\left(\mathbb{P}^{p}, \mathcal{O}_{\mathbb{P}^{p}}(d)\right)$ and since $H^{1}\left(\mathbb{P}^{p}, \mathcal{O}_{\mathbb{P}^{p}}(d)\right)=0$ there exists at least one current $S$ with $\bar{\partial} S=T$ by Theorem 6.1. For any other solution $S^{\prime}$ we have $\bar{\partial}\left(S-S^{\prime}\right)=0$ and the result follows from the regularity of distributions in $\operatorname{ker}(\bar{\partial})$.

$\S$ 8. Curves in $\mathbb{P}^{2}$. Let $\gamma \subset \mathbb{P}^{2}$ be a finite disjoint union of oriented closed curves of class $C^{1}$, and suppose $\gamma$ is the (current) boundary of a holomorphic 1-chain $T$. Choose a point $\mathbb{P}^{0} \notin \operatorname{supp}(T)$ and consider the projection

$$
\Omega \equiv \mathbb{P}^{2}-\mathbb{P}^{0} \stackrel{\pi}{\rightarrow} \mathbb{P}^{1}
$$

defined in $\S 7$. Recall that $\pi$ is isomorphic to the line bundle $\pi: \mathcal{O}(1) \rightarrow \mathbb{P}^{1}$, and so $\pi^{*} \mathcal{O}(1)$ has a tautological cross-section which we denote by $w$. Thus, for each $d \geq 0$ the current $\pi_{*}\left(w^{d} T\right)$ is a generalized section of $\mathcal{O}_{\mathbb{P}^{1}}(d)$ which satisfies the equation

$$
\bar{\partial} \pi_{*}\left(w^{d} T\right)=\pi_{*}\left(w^{d} \gamma\right)^{0,1}
$$

In particular, $\pi_{*}\left(w^{d} T\right)$ is a holomorphic section of $\mathcal{O}(d)$ over $\mathbb{P}^{1}-\gamma$.

Recall that $T$ is said to be positive over a point $a \in \mathbb{P}^{1}-\pi(\gamma)$ if its restriction to $\pi^{-1}(U)$ is positive for some neighborhood $U$ of $a$. From Theorem 5.3 we know that: 
Lemma 8.1. If $T$ is positive over a point $a \in \mathbb{P}^{1}-\pi \gamma$, then the holomorphic sections $\left\{\pi_{*}\left(w^{d} T\right)\right\}_{d=0}^{\infty}$ form a Newton hierarchy of level $\ell$ in a neighborhood of a where $\ell=\pi_{*}(T)=$ the sheeting number of $T$ at $a$.

We now look in the converse direction. Let $\gamma \subset \mathbb{P}^{2}$ be as above and ask whether $\gamma$ bounds a holomorphic 1-chain in $\mathbb{P}^{2}$. If it does, then it bounds infinitely many such chains since we are free to add any chain $S$ with $d S=0$. To eliminate this ambiguity we first fix the homology class $u \in H_{2}\left(\mathbb{P}^{2}, \gamma ; \mathbb{Z}\right)$ of the solution $T$. We assume $\partial u=[\gamma]$, and so by the short exact sequence: $0 \longrightarrow H_{2}\left(\mathbb{P}^{2} ; \mathbb{Z}\right) \longrightarrow$ $H_{2}\left(\mathbb{P}^{2}, \gamma ; \mathbb{Z}\right) \stackrel{\partial}{\rightarrow} H_{1}(\gamma ; \mathbb{Z}) \longrightarrow 0$, the class $u$ is determined by its intersection with any projective line $L$ which does not meet $\gamma$. In particular, for $a \in \mathbb{P}^{1}-\pi \gamma$ we set $L_{a}=\pi^{-1}(a)$ and note that

$$
L_{a} \cdot u=\pi_{*} u(a)=\text { the local net sheeting number of } T \text { over } a \text {. }
$$

If $d T=\gamma$, then $\pi_{*} T$ is an integer-valued function whose value at $a \in \mathbb{P}^{1}-\pi \gamma$ is precisely the net sheeting number of $T$ over $a$. It satisfies the equation $d\left(\pi_{*} T\right)=$ $\pi_{*} \gamma$, or equivalently $\bar{\partial}\left(\pi_{*} T\right)=\left(\pi_{*} \gamma\right)^{0,1}$ and is uniquely determined by this equation up to an integer constant. Choosing this constant is equivalent to choosing the homology class $u$.

Unfortunately, if there exists one solution in a given homology class, there exist infinitely many in that class, since one can add algebraic cycles homologous to zero. This indeterminacy is greatly reduced if we require $T$ to be positive over a chosen point $a \in \mathbb{P}^{1}-\pi \gamma$. Then there can exist at most a finite-dimensional family of solutions. Moreover, if such solutions exist for some $\ell>0$, then there is a minimal $\ell_{0} \geq 0$ where the problem is solvable, and for $\ell_{0}$ the solution $T$ is unique. For each $\ell>\ell_{0}$ all solutions are of the form $T+S$ where $S$ is in the finite-dimensional family of positive algebraic 1 -cycles homologous to $\left(\ell-\ell_{0}\right) \mathbb{P}^{1}$.

With this motivation we fix a point $a \in \mathbb{P}^{1}-\pi \gamma$ and an integer $\ell \geq 0$, and we look for holomorphic 1 -chains $T$ with $d T=\gamma$ which are positive and $\ell$-sheeted over $a$.

To begin, observe that since $d \gamma=0$, the currents $\pi_{*}\left(w^{d} \gamma\right)^{0,1}$ for $d \geq 0$ satisfy

$$
\bar{\partial} \pi_{*}\left(w^{d} \gamma\right)^{0,1}=0 \quad \text { on } \quad \mathbb{P}^{1}
$$

Therefore, by Proposition 7.2 the space

$$
\mathcal{T}_{d, \gamma} \stackrel{\text { def }}{=}\left\{C_{d} \in \mathcal{E}^{\prime 0}\left(\mathbb{P}^{1}, \mathcal{O}(d)\right): \bar{\partial} C_{d}=\pi_{*}\left(w^{d} \gamma\right)^{0,1}\right\} \quad \text { has dimension } d+1
$$

Any two elements of $\mathcal{T}_{d, \gamma}$ differ by a unique element of $H^{0}\left(\mathbb{P}^{1}, \mathcal{O}(d)\right)$, i.e., a homogeneous polynomial of degree $d$ in homogeneous coordinates $\left[z_{0}: z_{1}\right]$ on $\mathbb{P}^{1}$. This means the following. Let $z$ be an affine coordinate on $\mathbb{P}^{1}$ with $z(a)=0$, choose a trivialization of $\mathcal{O}(1)$ near 0 and for $C_{d} \in \mathcal{T}_{d, \gamma}$ consider the power series expansion

$$
C_{d}(z) \cong \sum_{k=0}^{\infty} \alpha_{k}(d) z^{k}
$$




\section{Observation 8.3}

(1) The coefficients $\left\{\alpha_{k}(d)\right\}_{k=d+1}^{\infty}$ are independent of the choice of $C_{d} \in \mathcal{T}_{d, \gamma}$ since any two elements in $\mathcal{T}_{d, \gamma}$ differ by a polynomial of degree $\leq d$ in $z$.

(2) There is a unique element $C_{d}^{o} \in \mathcal{T}_{d, \gamma}$ which vanishes to order $d$ at $a$.

To solve our problem we are seeking currents $C_{d} \in \mathcal{T}_{d, \gamma}$ which form a Newton heirarchy of level $\ell$ in a neighborhood of 0 (and are therefore of the form $\pi_{*}\left(w^{d} T\right.$ ) for some positive divisor of degree $\ell$ above that neighborhood). By Observation 8.3 there is some freedom in constructing these currents which comes from the polynomial ambiguity in $C_{d}$ for $d \leq \ell$. However, the equations governing Newton hierarchies precisely limit the possibilities and will lead to a series of non-linear moment conditions for the fixed coefficients $\alpha_{k}(d), k>d>\ell$. These will be necessary and sufficient for solving the problem.

The projection $\pi$ in (8.1) is called good if $\pi(\gamma)$ is an immersed curve with normal crossings. Such projections are generic.

Theorem 8.4. Let $\gamma \subset \mathbb{P}^{2}$ be a finite union of oriented closed curves of class $C^{1}$ in the complex projective plane. Choose a good projection $\pi: \mathbb{P}^{2}-\mathbb{P}^{0} \rightarrow \mathbb{P}^{1}$ from a point $\mathbb{P}^{0} \notin \gamma$ and a point $a \in \mathbb{P}^{1}-\pi \gamma$. Then $\gamma$ bounds a holomorphic 1-chain $T$ in $\mathbb{P}^{2}-\mathbb{P}^{0}$ which is positive over the point a with sheeting number $\ell$ if and only if there exists a sequence of homogeneous polynomials $\left\{p_{d}\left(z_{0}, z_{1}\right)\right\}_{d=1}^{\infty}$ with $\operatorname{deg}\left(p_{d}\right)=d$, such that $\left\{C_{d}^{o}+p_{d}\right\}_{d=1}^{\infty}$ is a Newton hierarchy of level $\ell$ in a neighborhood of $a$.

Proof. The necessity of the condition has been established. For the converse suppose $\left\{C_{d}^{o}+p_{d}\right\}_{d=1}^{\infty}$ is a Newton hierarchy of level $\ell$ in a neighborhood $U_{0}$ of $a$. By analyticity and Theorem 4.6 this must hold throughout the connected component $U$ of $a$ in $\mathbb{P}^{1}-\pi(\gamma)$. Hence, by Theorem 5.3 there is a positive holomorphic chain $\mathcal{T}$ in $\pi^{-1}(U)$ (a multisection of degree $\ell$ ) such that

$$
C_{d}=\pi_{*}\left(w^{d} \mathcal{T}\right) \quad \text { in } \quad U
$$

The remainder of the proof now follows precisely the arguments given in $\left[\mathrm{HL}_{1}\right.$, $\S 6]$. In $\left[\mathrm{HL}_{1}\right]$ we assumed $C_{d} \equiv 0$ in $U=$ the component of $\infty \in \mathbb{P}^{1}-\gamma$. Using boundary regularity, the "jump condition" $\left[\mathrm{HL}_{1}\right.$, Lemma 5.5] and the Hadamard criteria for rationality $\left[\mathrm{HL}_{1}\right.$, Theorem 4.6] we showed that for every connected component $V$ of $\mathbb{P}^{1}-\gamma$ there exists a holomorphic 1-chain $\mathcal{T}_{V}$ in $V \times \mathbb{C}$ such that

$$
\pi_{*}\left\{w^{d} \mathcal{T}_{V}\right\}=C_{d} \quad \text { in } V
$$

Furthermore, these chains extend across $\pi^{-1}(\gamma)$ to define a holomorphic 1-chain $\mathcal{T}$ with $d \mathcal{T}=\gamma$. All this was accomplished using just one good projection.

The arguments of $\left[\mathrm{HL}_{1}, \S 6\right]$ are inductive - from component to component of $\mathbb{P}^{1}-\gamma$. To begin one assumed $\mathcal{T}_{U}=0$. However, the arguments apply equally well if one merely assumes the existence of a holomorphic chain $\mathcal{T}$ in $\pi^{-1}(U)$ which satisfies (8.6) in $U$.

An alternative argument for the end of the proof can be given as follows. Let $\Delta_{\epsilon} \subset U$ be the disk of radius $\epsilon$ centered at $a$ and let $\mathcal{T}_{\epsilon}$ denote the restriction of 
$\mathcal{T}$ to $\pi^{-1}\left(\Delta_{\epsilon}\right)$. Choose $\epsilon$ so that $d \mathcal{T}_{\epsilon} \equiv \gamma_{\epsilon}$ is a regular (oriented, analytic) curve. Consider the new "boundary" curve $\Gamma=\gamma-\gamma_{\epsilon}$. Then for each $d$ we have by (8.6) that

$$
\widetilde{C}_{d} \stackrel{\text { def }}{=} C_{d}-\pi_{*}\left\{w^{d} \mathcal{T}_{\epsilon}\right\} \equiv 0 \quad \text { in } \Delta_{\epsilon},
$$

and using (8.2) we see that

$$
d \widetilde{C}_{d}=\pi_{*}\left\{w^{d} \Gamma\right\}
$$

We now pass to the affine coordinate chart $\zeta=z_{0} / z_{1}$ on $\mathbb{P}^{1}$ and the canonical trivialization given by $z_{1}$ over this chart. In this chart the solution to $\bar{\partial} \phi=\pi_{*}\left(w^{d} \Gamma\right)$ which vanishes to order $d$ at infinity is given explicitly by the Cauchy kernel, i.e.,

$$
\widetilde{C}_{d}(\zeta)=\frac{1}{2 \pi i} \int_{\Gamma} \frac{w^{d}}{\eta-\zeta} d \eta=\sum_{k=0}^{\infty}\left\{\frac{1}{2 \pi i} \int_{\Gamma} w^{d} \eta^{k} d \eta\right\} \zeta^{-k-1} \equiv 0
$$

in a neighborhood of infinity in the $\zeta$-plane. It follows that

$$
\int_{\Gamma} w^{d} \eta^{k} d \eta=0 \quad \text { for all } k, d \geq 0 .
$$

This restricted moment condition is the only hypothesis made in $\left[\mathrm{HL}_{1}, \S 6\right]$ and so those arguments apply directly to prove the existence of a holomorphic 1-chain $\widetilde{\mathcal{T}}$ with compact support in $\mathbb{C}^{2}$ and $d \widetilde{\mathcal{T}}=\Gamma$. Hence, $\mathcal{T} \equiv \widetilde{\mathcal{T}}+\mathcal{T}_{\epsilon}$ is a holomorphic 1-chain in $\mathbb{P}^{2}-\Gamma$ with $d \mathcal{T}=\gamma-\gamma_{\epsilon}+\gamma_{\epsilon}=\gamma$. Since $\mathcal{T}_{\epsilon}$ extends holomorphically across $\gamma_{\epsilon}$, it follows easily that $\mathcal{T}$ is a is a holomorphic chain in $\mathbb{P}^{2}-\gamma$ as desired.

By applying 4.6 we can restate Theorem 8.4 as follows. Let $\mathcal{H}(d) \equiv H^{0}\left(\mathbb{P}^{1}, \mathcal{O}_{\mathbb{P}^{1}}(d)\right)$ denote the space of homogeneous polynomials of degree $d$ in two variables.

Theorem 8.5. Let $\gamma, \mathbb{P}^{0}, \pi$ and $a$ be as in Theorem 8.4. Then $\gamma$ bounds a holomorphic 1-chain $T$ in $\mathbb{P}^{2}-\mathbb{P}^{0}$ which is positive over a with sheeting number $\ell$ if and only if there exist homogeneous polynomials $p_{d} \in \mathcal{H}(d)$ for $d=1, \ldots, \ell$, such that

$$
C_{k}^{o} \equiv Q_{k, \ell}\left(C_{1}^{o}+p_{1}, \ldots, C_{\ell}^{o}+p_{\ell}\right) \bmod \mathcal{H}(k)
$$

in a neighborhood of a for all $k>\ell$.

The non-linear moment conditions (8.9) represent an explicit sequence of polynomial relations among the coefficients $\left\{\alpha_{k}(d)\right\}_{k=d+1}^{\infty}$ discussed in 8.3 above.

Note 8.6. Theorems 8.4 and 8.5 continue to hold if one allows integer multiplicities on the connected components of $\gamma$. The proofs are essentially the same.

\$9. Curves in $\mathbb{P}^{n}$. Let $\gamma \subset \mathbb{P}^{n}$ be an embedded finite union of oriented closed curves of class $C^{1}$. Fix a codimension-2 linear subspace $\mathbb{P}^{n-2}$ with $\gamma \cap \mathbb{P}^{n-2}=\emptyset$ and consider the projection

$$
\Pi: \mathbb{P}^{n}-\mathbb{P}^{n-2} \rightarrow \mathbb{P}^{1}
$$


defined in $\S 7$. This projection is called good if $\left.\Pi\right|_{\gamma}$ is an immersion with normal crossings. Recall from $\S 7$ that $\Pi$ is isomorphic the vector bundle given as the $(n-1)$-fold direct sum

$$
\Pi: \mathcal{O}_{\mathbb{P}^{1}}(1) \oplus \cdots \oplus \mathcal{O}_{\mathbb{P}^{1}}(1) \longrightarrow \mathbb{P}^{1}
$$

Let $\mathbf{w}=\left(w_{1}, \ldots, w_{n-1}\right)$ denote the tautological cross section of $\Pi^{*}\left\{\mathcal{O}_{\mathbb{P}^{1}}(1) \oplus \cdots \oplus\right.$ $\left.\mathcal{O}_{\mathbb{P}^{1}}(1)\right\}$, where $w_{j}$ is the tautological section of the $j^{\text {th }}$ factor.

For each $\alpha=\left(\alpha_{1}, \ldots, \alpha_{n-1}\right) \geq(0, \ldots, 0)$ in $\mathbb{Z}^{n-1}$ consider the equation of $\mathcal{O}_{\mathbb{P}^{1}}(d)$ valued currents on $\mathbb{P}^{1}$ :

$$
\bar{\partial} C_{\alpha}=\Pi\left(w^{\alpha} \gamma\right)^{0,1}
$$

by $\S 7$ this equation has solutions unique up to holomorphic sections of $\mathcal{O}_{\mathbb{P}^{1}}(d)$.

Fix a point $a \in \mathbb{P}^{1}-\Pi(\gamma)$ and let $C_{\alpha}^{o}$ be the unique solution of (9.2) which vanishes to order $d$ at $a$. Then the general solution of (9.2) is of the form

$$
C_{\alpha}=C_{\alpha}^{o}+p_{\alpha}
$$

where $p_{\alpha} \in H^{0}\left(\mathbb{P}^{1}, \mathcal{O}(d)\right)$ is a homogeneous polynomial of degree $d$ in homogeneous coordinates on $\mathbb{P}^{1}$. We recall from $\S 5$ that this set of moments $\left\{C_{\alpha}\right\}_{\alpha \geq 0}$ corresponds to a homogeneous polynomial family of distributional sections of $\mathcal{O}_{\mathbb{P}^{1}}^{-}(d)$

$$
\mathbf{C}_{d}(\lambda)=\sum_{|\alpha|=d}\left(\begin{array}{l}
d \\
\alpha
\end{array}\right) C_{\alpha} \lambda^{\alpha}
$$

which satisfy the equation

$$
\bar{\partial} \mathbf{C}_{d}(\lambda)=\Pi_{*}\left\{(\lambda \cdot \mathbf{w})^{d} \gamma\right\}^{0,1}
$$

for

$$
\lambda \cdot \mathbf{w} \equiv \sum_{j} \lambda_{j} w_{j}=\pi_{\lambda}^{*}(w)
$$

where $\pi_{\lambda}: \mathcal{O}_{\mathbb{P}^{1}}(1)^{\oplus(n-1)} \longrightarrow \mathcal{O}_{\mathbb{P}^{1}}(1)$ is the $\lambda$-projection and $w$ is the tautological cross-section of $\pi^{*} \mathcal{O}_{\mathbb{P}^{1}}(1)$ over $\mathcal{O}_{\mathbb{P}^{1}}(1)$. Recall from Definition 5.6 that $\left\{C_{\alpha}\right\}_{\alpha \geq 0}$ is called a Newton family of level $\ell$ (with coefficients in $\mathcal{O}_{\mathbb{P}^{1}}(1)^{\oplus(n-1)}$ ) if $\left\{\mathbf{C}_{d}(\lambda)\right\}_{d \geq 0}$ is a Newton heirarchy of level $\ell$ (with coefficients in $\mathcal{O}_{\mathbb{P}^{1}}(1)$ ) for all $\lambda \in \mathbb{C}^{q}$.

Theorem 9.1. Let $\gamma \subset \mathbb{P}^{n}$ be an embedded finite union of oriented closed curves of class $C^{1}$ with possible integer multiplicities on each component. Choose a good projection $\Pi: \mathbb{P}^{n}-\mathbb{P}^{n-2} \longrightarrow \mathbb{P}^{1}$ from a codimension-2 linear subspace $\mathbb{P}^{n-2} \subset$ $\mathbb{P}^{n}-\gamma$ and fix a point $a \in \mathbb{P}^{1}-\Pi(\gamma)$. Then $\gamma$ bounds a holomorphic 1-chain in $\mathbb{P}^{n}-\mathbb{P}^{n-2}$ which is positive over a with sheeting number $\ell$ if and only if there exist homogeneous polynomials $\left\{p_{\alpha}\right\}_{\alpha>0}$ with $p_{\alpha} \in H^{0}\left(\mathbb{P}^{1}, \mathcal{O}(|\alpha|)\right)$, such that the family $\left\{C_{\alpha}\right\}_{\alpha \geq 0}$ given in (9.2-3) is a Newton family of level $\ell$ in a neighborhood of $a$. 
Proof. If $\gamma=d T$ where $T$ is a holomorphic 1-chain in $\mathbb{P}^{n}-\mathbb{P}^{n-2}$ which is positive with degree $\ell$ over the point $a$, then by Theorem 5.6, the functions $\mathbf{C}_{d}(\lambda)=\Pi_{*}((\lambda$. $\mathbf{w})^{d} T$ ) form a Newton family of degree $\ell$ in a neighborhood of $a$. Furthermore, we have $\bar{\partial} \mathbf{C}_{d}(\lambda)=\left\{d \mathbf{C}_{d}(\lambda)\right\}^{0,1}=\left\{\Pi_{*}\left((\lambda \cdot \mathbf{w})^{d} d T\right)\right\}^{0,1}=\left\{\Pi_{*}\left((\lambda \cdot \mathbf{w})^{d} \gamma\right)\right\}^{0,1}$. Expanding as in (9.4) gives the desired $\left\{C_{\alpha}\right\}_{\alpha \geq 0}$.

Conversely, suppose that there exist polynomials $\left\{p_{\alpha}\right\}_{\alpha \geq 0}$ such that the solutions (9.3) form a Newton family of level $\ell$ over a neighborhood of $a$. Then by Theorem 8.3 for almost every $\lambda$ the projection $\pi_{\lambda}(\gamma)$ bounds a holomorphic chain $T(\lambda)$ in $\mathbb{P}^{2}-\mathbb{P}^{0}$ with $d T(\lambda)=\pi_{\lambda}(\gamma)$. The holomorphic chain $T$ is then easily constructed from these projections as in $\left[\mathrm{HL}_{1}, \S 7\right]$.

An alternative argument for the second half of the proof can be given as follows. By Theorem 5.7 the existence of the Newton family $\left\{C_{\alpha}\right\}_{\alpha \geq 0}$ implies the existence of a multi-section $T_{\Delta}$ of degree $\ell$ over a disk-neighborhood $\Delta$ of $a$ in $\mathbb{P}^{1}-\Pi(\gamma)$ such that $\Pi_{*}\left\{w^{\alpha} T_{\Delta}\right\}=C^{\alpha}$ in $\Delta$. Now by shrinking $\Delta$ slightly we may assume that $d T_{\Delta}$ is a finite set of regular oriented closed curves with positive integer multiplicities. One now verifies, as in $\S 8$ above, that the curve $\gamma-d T_{\Delta}$ satisfies the moment condition of $\left[\mathrm{HL}_{1}\right]$ in $\mathbb{C}^{n+1}=\mathbb{P}^{n+1}-\overline{\Pi^{-1}(a)}$. Hence, by $\left[\mathrm{HL}_{1}, \S 7\right]$ there exists a holomorphic 1-chain $T_{0}$ with compact support in $\mathbb{C}^{n+1}$ such that $d T_{0}=\gamma-d T_{\Delta}$. Thus, $d T=\gamma$ where $T \equiv T_{0}+T_{\Delta}$ is easily seen to be a holomorphic chain in $\mathbb{P}^{n+1}-\gamma\left(\right.$ since $T_{\Delta}$ has an analytic continuation across $\left.d T_{\Delta}\right)$.

$\S 10$. Boundaries of Higher Dimension. Let $M \subset \mathbb{P}^{n}$ be a compact oriented embedded $(2 p-1)$-dimensional submanifold which is maximally complex. Fix a linear subspace $\mathbb{P}^{n-p-1}$ of complex codimension $p+1$ such that $M \cap \mathbb{P}^{n-p-1}=\emptyset$, and consider the projection

$$
\Pi: \mathbb{P}^{n}-\mathbb{P}^{n-p-1} \rightarrow \mathbb{P}^{p}
$$

defined in $\S 7$. This projection is called good if $\left.\Pi\right|_{M}$ is an immersion with normal crossings outside a $C^{1}$ stratified subset $\mathcal{S}=\bigcup_{\mu \geq 1} \mathcal{S}_{\mu}$ where $\operatorname{codim}_{\mathbb{R}}\left(\mathcal{S}_{\mu}\right)=2 \mu^{2}$. If $M$ is of class $C^{2 p-1}$, then by Theorem A.4 in [HL $\mathrm{HL}_{1}$ ] a generic projection is good.

As in $\S 9$ we note that $\Pi$ is isomorphic to $\Pi: \mathcal{O}_{\mathbb{P}^{p}}(1) \oplus \cdots \oplus \mathcal{O}_{\mathbb{P}^{p}}(1) \longrightarrow \mathbb{P}^{p}$ and let $\mathbf{w}=\left(w_{1}, \ldots, w_{n-1}\right)$ denote the tautological cross section of $\Pi^{*}\left\{\mathcal{O}_{\mathbb{P}^{p}}(1) \oplus \cdots \oplus\right.$ $\left.\mathcal{O}_{\mathbb{P}^{p}}(1)\right\}$. For $\alpha=\left(\alpha_{1}, \ldots, \alpha_{n-p}\right) \geq(0, \ldots, 0)$ in $\mathbb{Z}^{n-p}$ consider the equation

$$
\bar{\partial} C_{\alpha}=\Pi\left(w^{\alpha} M\right)^{0,1} .
$$

of $\mathcal{O}_{\mathbb{P}^{p}}(d)$-valued currents on $\mathbb{P}^{p}$. For $p>1$ the maximal complexity of $M$ implies that

$$
\bar{\partial} \Pi\left(w^{\alpha} M\right)^{0,1}=0
$$

(cf. $\left.\left[\mathrm{HL}_{1}, \S 3\right]\right)$. It follows that equation (10.2) has solutions unique up to holomorphic sections of $\mathcal{O}_{\mathbb{P} p}(d)$.

We now fix a point $a \in \mathbb{P}^{p}-\Pi(M)$ and let $C_{\alpha}^{o}$ be the unique solution of (10.2) which vanishes to order $d$ at $a$. The general solution of (10.2) is then of the form

$$
C_{\alpha}=C_{\alpha}^{o}+p_{\alpha}
$$

for $p_{\alpha} \in H^{0}\left(\mathbb{P}^{p}, \mathcal{O}(d)\right)$. 
Theorem 10.1. Let $M \subset \mathbb{P}^{n}$ be as above and suppose $\Pi: \mathbb{P}^{n}-\mathbb{P}^{n-p-1} \longrightarrow \mathbb{P}^{p}$ is a good projection from a linear subspace $\mathbb{P}^{n-p-1} \subset \mathbb{P}^{n}-M$. Fix a point $a \in$ $\mathbb{P}^{1}-\Pi(M)$. Then $M$ bounds a holomorphic $p$-chain in $\mathbb{P}^{n}-\mathbb{P}^{n-2}$ which is positive over $a$ with sheeting number $\ell$ if and only if there exist homogeneous polynomials $\left\{p_{\alpha}\right\}_{\alpha>0}$ with $p_{\alpha} \in H^{0}\left(\mathbb{P}^{1}, \mathcal{O}(|\alpha|)\right)$, such that the family $\left\{C_{\alpha}\right\}_{\alpha \geq 0}$ given in (10.2-3) is a Newton family of level $\ell$ in a neighborhood of $a$.

Proof. Necessity is proved exactly as in the proof of 9.1. The arguments for sufficiency in the proof of Theorems 8.1 and 9.1 (using results in $\left[\mathrm{HL}_{1}, \S 5,6\right]$ ) establish the existence of a holomorphic $p$-chain $T$ with $d T=M$ in $\mathbb{P}^{n}-M-\Pi^{-1}(\Pi \mathcal{S})$.

Furthermore, we have the following. let $U$ be the neighborhood of $a$ in $\mathbb{P}^{p}$ where $\left\{C_{\alpha}\right\}_{\alpha \geq 0}$ is a Newton family of level $\ell$, and suppose $L \subset \mathbb{P}^{p}$ is a projective line which meets $U$ and for which the linear subspace $\mathbb{P}_{L}^{n-p+1} \equiv \overline{\Pi^{-1}(L)}$ meets $M$ transversely. The restriction of $\left\{C_{\alpha}\right\}_{\alpha \geq 0}$ to $U \cap L$ is still a Newton family of level $\ell$. Furthermore, slicing by $L$ commutes with $\bar{\partial}$. Hence by Theorem 9.1 the curve $M_{L}=M \cap \mathbb{P}_{L}^{n-p+1}$ bounds a unique holomorphic chain $T_{L}$ with compact support in $\mathbb{P}_{L}^{n-p+1}-\mathbb{P}^{n-p-1}$ which is positive and $\ell$-sheeted over $a$. Moreover,

$$
T_{L}=T \cap \mathbb{P}_{L}^{n-p+1} \text { outside } \Pi^{-1}(\Pi \mathcal{S})
$$

since they give rise to the same Newton family in $U \cap L$. From this one concludes that $T$ has finite mass and bounded support in $\mathbb{P}^{n}-\mathbb{P}^{n-p-1}$. Consequently in $\mathbb{P}^{n}-\mathbb{P}^{n-p-1}$ we have

$$
d T=M+R \quad \text { with } \quad \operatorname{supp}(R) \subset \subset \Pi^{-1}(\Pi \mathcal{S})
$$

Now for each $\lambda \in \mathbb{C}^{n-p}$ let $\pi_{\lambda}$ and $\pi$ denote the projections defined in 5.5 with $\Pi=\pi \circ \pi_{\lambda}$. Then $\pi_{\lambda *} T$ is a holomorphic $p$-chain with $d \pi_{\lambda *} T=\pi_{\lambda *} M+R_{\lambda}$ where $R_{\lambda}=\pi_{\lambda *} R$ satisfies

$$
\operatorname{supp}\left(R_{\lambda}\right) \subset \pi_{\lambda}\left(\Pi^{-1} \Pi(\mathcal{S})\right)=\coprod_{\mu \geq 1} \pi^{-1}\left(\Pi \mathcal{S}_{\mu}\right)
$$

The current $R_{\lambda}$ is $d$-closed, integrally flat and of dimension $2 p-1$. Since $\pi^{-1}(\Pi \mathcal{S})$ is a $C^{1}$-stratified set of dimension $2 p-1$, arguments of [F, 4.1.15] show that $R_{\lambda}=$ $c \pi^{-1}(\Pi \mathcal{S})$ for an integer-valued function $c$ which is constant on components of the top strata of $\pi^{-1}(\Pi \mathcal{S})$. Unless $c=0$ this current has unbounded support in $\mathbb{P}^{p+1}-\mathbb{P}^{p-1}=\mathcal{O}_{\mathbb{P}^{p}}(1)$ because it is invariant under dilations in this bundle. This contradicts the boundedness of $T$ in $\mathbb{P}^{n}-\mathbb{P}^{n-p-1}$. Thus $R=0$ and the proof is complete.

$\S 11$. The General Result in Terms of Moment Conditions. The characterization given in Theorems 8.1,9.1 and 10.1 can be reexpressed as a countable family of non-linear moment conditions. They involve the spaces $\mathcal{H}(d) \equiv H^{0}\left(\mathbb{P}^{p}, \mathcal{O}(d)\right)$, and the universal polynomials $Q_{\alpha, \ell}$ given in Proposition 5.8. 
Theorem 11.1. Let $M \subset \mathbb{P}^{n}$ be a compact oriented submanifold of dimension $2 p-1$ which is maximally complex. Suppose $\Pi: \mathbb{P}^{n}-\mathbb{P}^{n-p-1} \longrightarrow \mathbb{P}^{p}$ is a good projection from a linear subspace $\mathbb{P}^{n-p-1} \subset \mathbb{P}^{n}-M$. Fix a point $a \in \mathbb{P}^{1}-\Pi(M)$. Then $M$ bounds a holomorphic p-chain in $\mathbb{P}^{n}-\mathbb{P}^{n-2}$ which is positive over a with sheeting number $\ell$ if and only if there exist homogeneous polynomials $p_{\alpha} \in \mathcal{H}(|\alpha|)$ for $0<|\alpha| \leq \ell$, such that the canonical solutions $C_{\alpha}^{o}$ of the equation (10.2) satisfy

$$
C_{\alpha}^{o} \equiv Q_{\alpha, \ell}(C) \quad \bmod \mathcal{H}(|\alpha|) \quad \text { for all } \quad|\alpha|>\ell
$$

in a neighbornood of $a$, where $C_{\alpha}=C_{\alpha}^{o}+p_{\alpha}$ for $|\alpha| \leq \ell$.

As discussed in $\S 3$, when $p=1$ the equations (11.1) reduce to certain universal polynomial relations among the classical moments of the curve in an affine chart on $\mathbb{P}^{n}$.

§12. The Relation to Dolbeault-Henkin. The main results here are related and complementary to those of P. Dolbeault and G. Henkin [D], $\left[\mathrm{DH}_{1,2}\right]$. In our notation these authors consider the canonical solutions $C_{\alpha}^{o}$ for $|\alpha| \leq 1$ as functions of the projection $\Pi$. That is, they allow the projection to vary in a neighborhood $U$ of some fixed $\Pi_{0}$ in the Grassmannian and consider the $C_{\alpha}^{o}$ as functions on $U$. Their main result asserts that $\alpha$ bounds a holomorphic $p$-chain in $\mathbb{P}^{n}$ iff the $C_{\alpha}^{o}$ can be expressed as a finite linear combination of functions which satisfy a certain non-linear shock equation on $U$.

This result is beautiful and quite general in that no positivity assumptions are required. On the other hand it is somewhat mysterious in that the functions which satisfy the shock equation are produced deus ex machina. There is no direct relationship to the geometry of the boundary $\gamma$.

In our result we fix one projection $\Pi_{0}$ and give a set of explicit congruences for the functions $C_{\alpha}^{o}$ (for all $\alpha$ ) which are equivalent to the existence of the holomorphic

$p$-chain. For this we need a positivity condition which guarantees at most a finitedimsnional family of possible solutions.

\section{References}

[[A]] H. Alexander, Linking and holomorphic hulls, J. Diff. Geom. 38 (1993), 151-160.

[[AW 1$]]$ H. Alexander and J. Wermer, Several Complex Variables and Banach Algebras, Springer-Verlag, New York, 1998.

$\left[\left[\mathrm{AW}_{2}\right]\right] \mathrm{H}$. Alexander and J. Wermer, Linking numbers and boundaries of varieties, Ann. of Math. 151 (2000), 125-150.

[[B]] S. Bochner, Analytic and meromorphic continuation by means of Green's formula, Ann. of Math. 44 (1943), 652-673.

[[deR]] G. de Rham, Variétés Différentiables, formes, courants, formes harmoniques, Hermann, Paris, 1955. 
[[D]] P. Dolbeault, On holomorphic chains with given boundary in $\mathbb{C P}^{n}$, Springer Lecture Notes, no. 1089, (1983), 1135-1140.

$\left[\left[\mathrm{DH}_{1}\right]\right]$ P. Dolbeault and G. Henkin, Surfaces de Riemann de bord donné dans $\mathbb{C P}^{n}$, Aspects of Math. 26 (1994), 163-187, Contributions to complex analysis and analytic geometry, Vieweg.

$\left[\left[\mathrm{DH}_{2}\right]\right]$ P. Dolbeault and G. Henkin, Chaines holomorphes de bord donné dans $\mathbb{C P}^{n}$, Bull. Soc. Math. de France 125 (1997), 383-445.

[[DL]] T.-C. Dinh and M. Lawrence, Polynomial hulls and positive currents, Ann. Fac. Sci de Toulouse 12 (2003), 317-334.

[[Fa 1$]]$ B. Fabré, Sur l'intersection d'une surface de Riemann avec des hypersurfaces algébriques, C. R. Acad. Sci. Paris 322 Série I (1996), 371-376.

$\left[\left[\mathrm{Fa}_{2}\right]\right]$ B. Fabré, On the support on complete intersection 0-cycles, The Journal of Geometric Analysis 12 (2002), 601-614.

[[F]] H. Federer, Geometric Measure Theory, Springer-Verlag, New York, 1969.

[[Fu]] W. Fulton, Intersection Theory, Springer-Verlag, New York, 1984.

[[H]] F.R. Harvey, Holomorphic chains and their boundaries, Several Complex Variables, Proc. of Symposia in Pure Mathematics XXX Part 1, A.M.S., Providence, RI, 1977, pp. 309-382.

$\left[\left[\mathrm{HL}_{1}\right]\right]$ F.R. Harvey and H.B. Lawson, Jr., On boundaries of complex analytic varieties, I, Ann. of Math. 102 (1975), 223-290.

[[HL $\left.\left.\mathrm{HL}_{2}\right]\right]$ F.R. Harvey and H.B. Lawson, Jr., On boundaries of complex analytic varieties, II, Ann. of Math. 106 (1977), 213-238.

$\left[\left[\mathrm{HL}_{3}\right]\right]$ F.R. Harvey and H.B. Lawson, Jr., Projective hulls and the projective Gelfand transformation, (to appear)..

$\left[\left[\mathrm{HL}_{4}\right]\right]$ F.R. Harvey and H.B. Lawson, Jr., On the Alexander-Wermer Theorem, (to appear)..

[[HS]] F.R. Harvey and B. Shiffman, A characterization of holomorphic chains, Ann. of Math. 99 (1974), 553-587.

[[Ho]] L. Hörmander, An Introduction to Complex Analysis in Several Variables, Van Nostrand, Princeton, N. J., 1966.

[[L]] M. Lawrence, Polynomial hulls of rectifiable curves, Amer. J. Math 117 (1995), 405-417.

[[S]] G. Stolzenberg, uniform approximation on smooth curves, Acta math. 115 (1966), 185-198.

[[W 1$]]$ J. Wermer, The hull of a curve in $\mathbb{C}^{n}$, Ann. of Math. 68 (1958), 550-561.

$\left[\left[\mathrm{W}_{2}\right]\right]$ J. Wermer, The argument principle and boundaries of analytic varieties, Operator Theory: Advances and Applications 127 (2001), 639-659. 